\title{
Expertengespräch: Immunsystem und Infekte
}

Wie geht die Ganzheitsmedizin mit Infektionen um und welche Methoden haben die verschiedenen Richtungen? Prophylaxe ist das primäre Ziel, und wenn es zu einer Erkrankung gekommen ist, muss der Körper unterstützt werden, die Infektion zu bekämpfen. Das ist der Konsens von Bernhard Uehleke, Matthias Rostock und Martin Frei-Erb, die wir als Vertreter der Kneipp-Medizin, der klassischen Homöopathie und der Phytotherapie zum Gespräch gebeten haben.

Warum wünschen sich Ihre Patienten eine ganzheitliche medizinische Behandlung?

Rostock: Meine Erfahrung ist, dass die Patienten, die bewusst mit ihrer Gesundheit umgehen, kritisch nach einem differenziert vorgehenden Therapeuten suchen: Einen Behandler, der kundig konventionelle Therapien einsetzt, wo es keine wirklichen Alternativen gibt, aber der auch mit der Naturheilkunde umzugehen weiss, wann immer sie aussichtsreich einzusetzen ist. Sie suchen einfach die optimale Therapie für sich und sind kritisch, aber dafür auch bereit, selbst Verantwortung zu übernehmen.

Frei-Erb: Die Patienten möchten einen ganzheitlichen Ansatz; sie möchten nicht nur eine Symptombehandlung, sondern dass man sie als Individuum ernst nimmt. Viele haben Bedenken wegen Nebenwirkungen der klassischen Medikamente, wollen häufig keine Antibiotika und möchten zuerst etwas anderes probieren.

Uehleke: Ich würde von einem komplementärmedizinischen bzw. von einem naturheilkundlichen Ansatz sprechen, der in meinem Fall auf dem Kneipp'schen Säulenmodell beruht. Nach dem Zweiten Weltkrieg war es notwendig, einen anderen Begriff als den der Biomedizin zu finden. In den USA wird von der integrativen Medizin gesprochen; bei uns hat sich der etwas unglückliche Begriff der Ganzheitsmedizin eingebürgert.

Welche Infektionen sind in Ihrem medizinischen Alltag am häufigsten und wie behandeln Sie sie?

Uehleke: Die häufigsten Infektionen bekommen wir als Ärzte gar nicht zu sehen. Das sind Atemwegsinfekte, wie Erkältungen und leichte Grippen, oder Harnwegsinfekte oder Infektionen mit Herpes. Diese werden von den Patienten meist selbst behandelt und kommen höchstens dann zur Sprache, wenn der Patient wegen etwas anderem zum Arzt kommt. Dann sind aber die Kneipp'schen Methoden ausserordentlich erfolgreich, besonders das Prinzip der allgemeinen Abhärtung. Kurze Kältereize sorgen dafür, dass das Immunsystem gestärkt und die periphere Durchblutung verbessert wird. Auch andere Methoden, die unter dem Begriff der «Hausmittel» zusammengefasst werden können, sind bei der Behandlung dieser häufigen Infekte meist gut wirksam.

Rostock: Wie bei anderen Methoden der Naturheilkunde geht es bei der Phytotherapie erst einmal darum, den
Patienten so zu unterstützen, dass seine Resistenzmechanismen stark genug sind, dass es gar nicht zu einer Erkrankung kommt. Wenn jemand einen Infekt hat, gibt es Mittel, die helfen, eine Infektion rascher zu überwinden, die Symptome zu lindern und das Risiko einer bakteriellen Superinfektion zu reduzieren.

Im Zusammenhang mit akuten Infekten muss man Echinacea nennen, bei der man gut gezeigt hat, dass die Wirkstoffe insbesondere zu Beginn einer Infektion helfen. Sie stimulieren körpereigene Abwehrmechanismen, die natürlich viel wirksamer sind, wenn sich die Keime erst kurz festgesetzt haben. Es gibt Doppelblindstudien, bei denen Echinacea gegenüber Placebo bei den ersten Infektanzeichen angewendet wurde. Bei der Echinacea-Gruppe war der Anteil der Menschen höher, die keine Infektion bekommen haben, und wenn sie krank wurden, war die Infektion schneller überwunden. Darüber hinaus gibt es unter anderem mehrere Studien aus den letzten Jahren, die den Erfolg von Pelargonium bei akuten Atemwegsinfektionen belegen.

Frei-Erb: Es gibt mittlerweile Studien, wie die von Steinsbekk, die zeigen, dass klassische Homöopathie bei Luftwegsinfekten gute Ergebnisse zeigt. Andere Untersuchungen, bei denen

\section{KARGER}

Fax +497614520714 


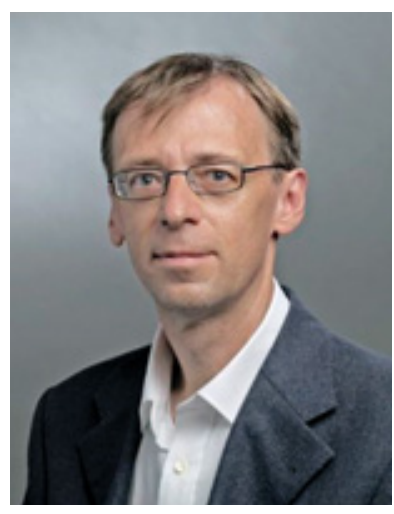

Dr. med. Martin Frei-Erb ist Facharzt FMH für Allgemeinmedizin, absolvierte eine Ausbildung in klassischer Homöopathie. Eröffnung einer Hausarztpraxis 1993 in Thun und seit 2008 Dozent für klassische Homöopathie an der Universität Bern und Mitarbeiter der KIKOM.

«Homöopathie ist bei der Versorgung von Luftwegsinfekten eine kostengünstige und wirksame Alternative. Man kann die häufigsten Infekte gut mit ihr behandeln.»

die Wirkung von homöopathischen Komplexmitteln getestet wurde, zeigen, dass auch diese Präparate gut wirken. Eine Studie von Haidvogl zum Einsatz der Homöopathie bei der Versorgung von Luftwegsinfekten belegt, dass sie eine kostengünstige und wirksame Alternative zur Schulmedizin ist. Man kann die häufigsten Infekte gut mit der Homöopathie behandeln.

In der letzten Zeit mehren sich die Hinweise, dass "unhygienische» Bedingungen während der Kindheit und Jugend das Immunsystem stärken.

Frei-Erb: Ich würde nicht von unhygienischen Bedingungen sprechen, sondern von zu viel Hygiene, die das Immunsystem schwächen kann bzw. dafür sorgt, dass es sich nicht richtig entwickeln und stark werden kann. Es kann nicht "trainieren», und ich habe den Eindruck, dass die fehlenden Antigene auch dazu führen, dass es vermehrt zu allergischen Reaktionen kommt. Im Erwachsenenalter kann man es aber mit homöopathischen Konstitutionsmitteln wieder herstellen und stärken.

Uehleke: Da ist wahrscheinlich etwas dran, dass die verwöhnte Jugend heute ein etwas schlechteres Immunsystem hat als noch vor einer Generation. Kinder und Jugendliche wachsen einfach unter komfortableren Bedingungen auf und sind weniger abgehärtet. Es gibt Hinweise, dass Stallluft einen positiven Einfluss auf das Immunsystem hat und dass die Allergene, die von Tieren kommen, dazu führen, dass das Immunsystem stärker sensibilisiert wird. Das Gleiche gilt auch für Erde, die scheinbar das Abwehrsystem stärkt, wenn sie in den Verdauungstrakt gerät. Das Thema ist aber komplexer und man darf es nicht auf die Hygiene reduzieren. Es spielen noch zwei andere Faktoren eine Rolle. Ich glaube, dass Kinder heute viel früher und häufiger verschiedenen Krankheitserregern gegenüber exponiert sind. Weil sie in einem frühen Alter in die Krippe gehen, sind sie ständig in Kontakt mit anderen Kindern und auch deren Erregern. Sie stehen, was die Belastung mit Krankheitserregern betrifft, unter Dauerstress. Und es kommt ein weiterer Faktor hinzu. Durch das frühere Abstillen kommen die Babys auch nicht mehr so lange mit dem mütterlichen Immunsystem in Kontakt und profitieren auch nicht mehr von der Schutzfunktion durch deren Immunzellen.

\section{Lässt sich das Immunsystem durch die verschiedenen Methoden der} Komplementärmedizin stärken und gibt es Untersuchungen, die das belegen?

Rostock: Ganz klar, ja. Der Schwerpunkt in der Komplementärmedizin liegt in der Prophylaxe und die Methoden der Naturheilkunde beginnen bei den banalen Dingen, die man leicht vergisst. Der erste Punkt ist die Ernährung. Sie muss vitalstoff- und vitaminreich sein. Besonders Vitamin $C$ ist wichtig, das nicht unbedingt über Brausetabletten eingenommen werden muss. Da bieten sich auch natürliche Quellen, an, wie etwa Sanddorn oder Hagebutte, die es als Saft oder Mousse gibt. Der zweite wichtige Punkt sind Bewegung und moderater Sport. Dadurch werden immunologische Mechanismen gefördert. Die Betonung liegt auf moderat, denn auch hier bestimmt die Dosis das Gift: Marathonläufer haben eine erhöhte Infektionsanfälligkeit. Der dritte Punkt sind genügend Erholungsphasen. Also ganz banal, ausreichend Schlaf, Pausen einhalten, die eigene Rhythmik beachten. Ausserdem sollte man, wenn möglich, Stress vermeiden, denn der schwächt die Resistenzmechanismen. Es gibt schon aus den 70er- und 80er-Jahren Studien, die zeigen, dass etwa Meditation einen positiven Einfluss hat.

Frei-Erb: Diesen Ansatz verfolgt auch die Homöopathie. Den Körper stärken, um die Infektion zu verhindern. Wir nennen das die konstitutionelle Behandlung. Daten dazu gibt es allerdings nur wenige, aber die Erfahrung zeigt, dass der Ansatz wirkt.

Uehleke: Was die Kneipp'schen Methoden betrifft, gibt es tatsächlich Studien, die einen positiven Effekt bei Atemwegsinfektionen zeigen. Bei einer Vorlaufzeit von 2-3 Monaten wirken zweimal pro Woche Sauna mit kalten Anwendungen vorbeugend. Der Wirkmechanismus, der dahinter steckt, ist durchaus plausibel. Die Kältereize bewirken eine Stressreaktion des Körpers. Es kommt zum Ausstoss von zum Beispiel Adrenalin und Cortison und das zelluläre Immunsystem reagiert darauf sehr stark. Kälte gehört zu den lebensbedrohlichsten Bedingungen, denen ein Körper ausgesetzt sein kann, und deshalb ist die sehr heftige Reaktion des Immunsystems auch verständlich. Neben der Immunantwort ist die bessere periphere Durchblutung wichtig bei der 


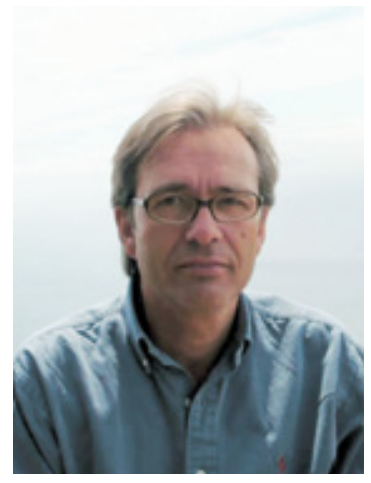

Dr. med. Matthias Rostock ist Facharzt für Innere Medizin, Hämatologie und Onkologie. Er ist als Assistenzarzt und Mitarbeiter am Institut für Naturheilkunde der Universität Zürich tätig.

"Die Kombination verschiedener naturheilkundlicher Methoden wie Ordnungstherapie, Hydrotherapie und Pflanzenheilkunde halte ich für sinnvoll, da sie sich gegenseitig ergänzen.»

Vorbeugung von Infektionen. Man weiss, dass Menschen, die kalte Hände oder Füsse haben, also bei denen die Durchblutung der Extremitäten nicht so gut ist, häufiger erkältet sind.

\section{Sehen Sie die Möglichkeit,} allergische Reaktionen durch eine Stimulation des Immunsystems zu behandeln?

Frei-Erb: Die homöopathischen Methoden wirken gut. Bei der Pollenallergie wird ausserhalb der Saison konstitutionell behandelt, um den Körper zu stärken. Er soll kräftig genug sein, um auf die Allergene nicht mit einer Immunreaktion zu antworten. In der Regel reicht diese konstitutionelle Behandlung. Wenn es aber doch zu einer allergischen Reaktion kommt, geben wir akut ein homöopathisches Ergänzungsmittel. Das Mittel, um die Konstitution zu fördern, ist von der Art der Allergie unabhängig; das Ergänzungsmittel richtet sich nach dem Krankheitsbild. Nach meiner Erfahrung ist die Behandlung mit der vergleichbar, die sich durch eine Sensibilisierung erzielen lässt.
Uehleke: Was sich aber gezeigt hat, ist, dass die Kneipp'schen Methoden hier helfen können. Obwohl sie das Immunsystem anregen und man sie deshalb eigentlich für kontraindiziert halten sollte, wirken sie in diesen Fällen beruhigend auf das Abwehrsystem. Eine ähnliche, auf den ersten Blick widersprüchliche Wirkung gibt es bei der Behandlung von niedrigem Blutdruck und Hypertonie mit der Kneipp'schen Methode. Sie scheint das Immunsystem wieder «einzuregeln».

Wo müssen aus Ihrer Sicht Methoden der Schulmedizin zum Einsatz

kommen bzw. wo ist die

Zusammenarbeit mit anderen

ganzheitlichen medizinischen

Disziplinen angebracht?

Uehleke: Die Kombination von schulmedizinischen und komplementärmedizinischen Methoden ist bislang weder systematisch erforscht worden noch wird sie von den Patienten auch gewünscht. Die Patienten wechseln oft von der Schulmedizin zu einem Verfahren der Naturheilkunde und wieder zurück. Sie probieren meist verschiedene Methoden der Behandlung aus. Ich habe auch generelle Zweifel, dass sich die Wirkung von zwei verschiedenen Methoden einfach addieren lässt - aber es gibt anderseits auch Beispiele, z.B. in der Onkologie, wo sich Naturheilmittel ergänzend zur Chemotherapie oder Bestrahlung bewährt haben.

Frei-Erb: Es kommt auf die Krankheit und den Schweregrad an und es geht darum, die ideale Therapie zu finden. Bei milden Infekten kann man nur homöopathisch behandeln, bei schweren Fällen greift man zusätzlich zu konventionellen Mitteln, auch zu Antibiotika. Wenn man zur Homöopathie greift, hat man als Arzt eine zusätzliche Behandlungsalternative. Allerdings kombiniere ich im Alltag andere naturheilkundliche und homöopathische Methoden selten. Der
Ansatz geht ja in die gleiche Richtung und zielt auf die Förderung der Lebenskräfte $a b$.

Rostock: Die Kombination verschiedener naturheilkundlicher Methoden, wie die Ordnungstherapie, Hydrotherapie und Phytotherapie, finde ich sehr sinnvoll, da sich viele gegenseitig ergänzen.

\section{Wo liegen aus der Sicht Ihres Ansatzes die grössten Herausforderungen, Infekte zu bekämpfen?}

Rostock: Ich würde gar nicht therapeutische Fragen in den Vordergrund stellen. In der Hausarztpraxis ist es die grosse Herausforderung, unter erheblichem Zeit- und Budgetdruck immer die richtige Entscheidung zwischen konventioneller und komplementärer Therapie zu fällen.

Uehleke: Im Moment mangelt es an Antworten der Naturheilkunde auf die Fragen, die die Entwicklung des Gesundheitswesens uns stellt. Das gilt ganz besonders für die Herausforderungen, die die immer älter werdende Gesellschaft mit sich bringt, mit der wir in der Zukunft konfrontiert sein werden. Ihr Einsatz in der Geriatrie, die in der Zukunft enorm wichtig wird, ist bisher vernachlässigt worden. Hier und auch im Allgemeinen gilt, dass es nach wie vor viel $\mathrm{zu}$ wenige Studien gibt, die sich mit der Wirksamkeit der verschiedenen Methoden beschäftigen. Hier muss die Naturheilkunde offensiver neuer Einsatzgebiete angehen. Die Wirksamkeit muss viel besser untersucht werden, so etwa von Phytopräparaten oder von Hausmitteln, die etwa bei Grippe seit langem angewendet werden, wie etwa Inhalationen oder warme und kalte Wickel. Aus der Erfahrung weiss man zwar, dass sie wirken, aber es fehlen in vielen Fällen einfach die Studien, die das auch wirklich zweifelsfrei belegen. 


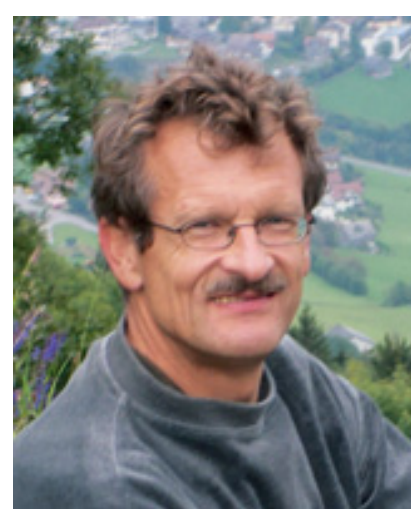

Dr. med. Dr. rer. nat. Bernhard Uehleke ist Assistenzarzt und Mitarbeiter am Institut für Naturheilkunde der Universität Zürich. Er betreut als Forschungskoordinator zudem den Bereich klinische Forschung der Klinik und Hochschulambulanz für Naturheilkunde der Charité in Berlin.

"Dort, wo es sich medizinisch rechtfertigen lässt, halte ich Verfahren der Naturheilkunde für die Methode der Wahl.»

Frei-Erb: In der Homöopathie ist der Ausbildungsstand der Ärzte entscheidend für die Anwendung und den Erfolg der Methode. Die Krankheitsbilder zu kennen und dann richtig reagieren zu können, ist von grosser Bedeutung. Bei $80 \%$ der Patienten kann man rasch das richtige Mittel finden. Es kommt auf die Erfahrung des homöopathischen Arztes an. Die KIKOM bietet den Berner Hausärzten die klassische Homöopathie als Weiterbildung an. Die Nachfrage von Laien ist sehr gross, von Ärzten allerdings leider geringer.

\section{Bei welchen Infekten haben Sie die besten Erfahrungen mit Ihrem Ansatz gemacht?}

Rostock: Die Phytotherapie kann bei verschiedenen Infekten der Atemwege, des Magen-Darm-Traktes und der ableitenden Harnwege mit Erfolg eingesetzt werden.

Uehleke: Ganz klar bei den Atemwegserkrankungen, und zwar sowohl bei der Prävention als auch bei der Behandlung. Hier haben sich zum Beispiel heisse Bäder, Kaltreize oder das Einreiben mit verschiedenen Balsamen bewährt. Man muss aber mit Standardempfehlungen vorsichtig sein, denn die Behandlung muss sich an den Symptomen orientieren, und die können sich im Verlauf der Erkrankung verändern, wie etwa bei einer Grippe. Jedes Symptom verlangt dann eine eigene Behandlung.

Frei-Erb: Mit der Homöopathie habe ich bei viralen Infekten, wie etwa Luftwegserkrankungen oder beginnenden Pneumonien, sehr gute Erfahrungen gemacht, die ja auch durch Untersuchungen bestätigt werden. Auch rezidivierende Blasenentzündungen oder Herpeserkrankungen lassen sich mit der klassischen Homöopathie erfolgreich behandeln.

Was ist in Ihrem medizinischen Alltag bei der Therapie von Infekten zu beachten? Wo liegen die Grenzen Ihres Ansatzes?

Frei-Erb: Wenn jemand mit einer bakteriellen Hirnhaut- oder Lungenentzündung kommt, muss man die auch als homöopathischer Arzt mit Antibiotika behandeln. Homöopathische Mittel kann man als Ergänzung und zur Unterstützung geben. Es gibt eine Untersuchung von Frass, die zeigt, dass bei ganz schweren Infekten, wie etwa der Sepsis, die Mortalität signifikant gesenkt werden kann, wenn zusätzlich zur konventionellen Behandlung homöopathische Mittel einsetzt werden.

Rostock: Die Grenzen liegen da, wo die Komplementärmedizin eine rasch wirkende Behandlung bei einem vital bedrohten Patienten nicht liefern kann, um einen bleibenden Schaden von ihm abzuwenden. Dazu gehört etwa auch die Therapie von schweren Lungenentzündungen oder des neutropen Fiebers bei Tumorpatienten unter Chemotherapie. Hier führt dann kein Weg an Antibiotika vorbei.

Gibt es Möglichkeiten, Autoimmunerkrankungen mit lhrem ganzheitlich medizinischen Ansatz wirkungsvoll zu behandeln?

Uehleke: Ja, wir haben solche Erkrankungen mit der Reiztherapie behandelt; es gibt auch Versuche, sie mit Eigenbluttherapien zu modulieren oder mit Omega-3-Fettsäuren weniger entzündungsbereit zu machen. Man muss sich aber auch vor Augen führen, dass Autoimmunerkrankungen ihre Ursache in einer gestörten Darmfunktion haben können. Das ist vom Wirkmechanismus her plausibel. Wenn die Darmflora nicht intakt ist, ist die Barrierefunktion des Darms nicht mehr gewährleistet und Eiweissbruchstücke treten in den Blutkreislauf ein und sorgen dort für eine Immunantwort, die etwa für die vielen Eiweissallergien verantwortlich sein könnten, die wir heute sehen.

Frei-Erb: Es gibt zwar keine Studien, aber ich persönlich habe den Eindruck, dass wir Autoimmunkrankheiten mit homöopathischen Methoden nur schwierig in den Griff bekommen können.

Rostock: Autoimmunerkrankungen erfordern in jedem Fall ein multimodales Vorgehen, in dessen Rahmen auch Phythotherapeutika wie zum Beispiel Weihrauchextrakt eingesetzt werden können.

\section{Wie stehen Sie zum Einsatz von Antibiotika?}

Uehleke: Sie sind da gerechtfertigt, wo sie eine Wirkung zeigen, die jener der Naturheilverfahren überlegen ist, und wo diese Wirkung schnell erzielt werden muss. Man muss aber sehen, dass Antibiotika heute viel zu schnell, bei 
der falschen Indikation und ohne Rücksicht auf die mögliche Resistenzentwicklung verschrieben werden. Dort, wo es sich medizinisch rechtfertigen lässt, halte ich Verfahren der Naturheilkunde für die Methode der Wahl.

Rostock: Es gibt Infektionen, wo man sie einsetzen muss, um den Patienten nicht zu gefährden. Hier sollte man zwischen Schulmedizin und Naturheilkunde keinen Glaubenskrieg austragen.

Frei-Erb: Antibiotika lehne ich als Homöopath nicht ab, denn jedes Medikament hat seinen berechtigten Einsatzbereich. Dass Antibiotika zu schnell, zu oft und häufig auch unnötig gegeben werden, ist klar. Bei einem schweren bakteriellen Infekt sind sie das Beste für den Patienten.

\section{Wie werden chronische Infekte durch Ihren Ansatz behandelt?}

Uehleke: Auch hier ist die Abhärtung das Mittel der Wahl. Der Organismus muss gestärkt sein, damit er mit diesen Keimen besser fertig wird.

Frei-Erb: Das gilt auch für die Homöopathie. Die Stärkung des Körpers steht auch hier im Mittelpunkt.
Wie sehen Sie die Behandlung von Chlamydieninfektionen durch die Ganzheitsmedizin?

Frei-Erb: Für viele Frauen sind sie ein Problem, das ich homöopathisch auch mit einer Konstitutionstherapie behandeln würde. Eine chronische Infektion zeigt, dass die Lebenskraft geschwächt ist und der Körper deshalb gestärkt werden muss. Bei Chlamydien würde ich aber auch den Partner behandeln.

Rostock: Chlamydieninfektionen im Genitalbereich sind oft asymptomatisch, können aber unter anderem in die Gebärmutter aufsteigen und zu Verklebungen des Eileiters und zu nachfolgender Sterilität führen. Antibiotikaresistenzen sind selten. Ich würde primär zu einer antibiotischen Therapie unter Einschluss des Sexualpartners raten und die Stärken der Naturheilkunde eher in der Nachbehandlung und Rezidivprophylaxe sehen.

Uehleke: Wenn es um die Behandlung von Harnwegsinfekten geht, helfen aus Sicht der Kneipp'schen Medizin die allgemeine Abhärtung und vor allem viel trinken. Das gilt ganz besonders für Frauen, die zu wenig Flüssigkeit zu sich nehmen. Sie sind nicht nur anatomisch für Harnwegsinfekte besonders anfällig, sondern zusätzlich auch durch den Flüssigkeitsmangel, den sie oft herbeiführen, um etwa öffentliche Toiletten zu meiden.

Ist die Behandlung von Infektionen, die durch antibiotikaresistente Stämme verursacht wurden, mit Ihrem Ansatz möglich?

Rostock: Diese Frage wird in der Arzneimittelforschung der letzten Jahre intensiv verfolgt und insbesondere $\mathrm{zu}$ verschiedenen ätherischen Ölen liegen bereits interessante Ergebnisse vor.

Uehleke: Hier sind die Kneipp'schen Methoden denen der Schulmedizin überlegen. Denn sie wirken bei resistenten Stämmen ebenso wie bei nicht resistenten. Hier liegt eine Chance, die die Komplementärmedizin nutzen kann, da wir in Zukunft mit einem vermehrten Auftreten solcher Stämme rechnen müssen.

Frei-Erb: Hier denkt man natürlich sofort an Tuberkulose oder an die Resistenzentwicklung in den Spitälern. Theoretisch wäre das ein Einsatzbereich für die Homöopathie, aber es gibt keine Daten und wahrscheinlich auch nur sehr wenig Erfahrung zu dem Thema. Es wäre interessant, wenn ein Akutspital den Einsatz der Homöopathie in solchen Fällen evaluieren würde.

Interview: Oliver Klaffke 